\title{
Evidence of defect band mechanism responsible for band gap evolution in $(\mathrm{ZnO})_{1-x}(\mathrm{GaN})_{x}$ alloys
}

\author{
V. S. Olsen $\odot,{ }^{1}$ G. Baldissera, ${ }^{2}$ C. Zimmermann, ${ }^{1}$ C. S. Granerød, ${ }^{1}$ C. Bazioti, ${ }^{1}$ A. Galeckas, ${ }^{1}$ B. G. Svensson, ${ }^{1}$ \\ A. Yu. Kuznetsov, ${ }^{1}$ C. Persson,,${ }^{1,2} \varnothing$. Prytz, ${ }^{1}$ and L. Vines ${ }^{1}$ \\ ${ }^{1}$ Department of Physics, Center for Materials Science and Nanotechnology, University of Oslo, \\ P. O. Box 1048, Blindern, N-0316 Oslo, Norway \\ ${ }^{2}$ Department of Materials Science and Engineering, KTH Royal Institute of Technology, SE-100 44 Stockholm, Sweden
}

(Received 20 May 2019; revised manuscript received 30 August 2019; published 4 October 2019)

\begin{abstract}
It is known that $(\mathrm{ZnO})_{1-x}(\mathrm{GaN})_{x}$ alloys demonstrate remarkable energy band bowing, making the material absorb in the visible range, in spite of the binary components being classical wide band gap semiconductors. However, the origin of this bowing is not settled; two major mechanisms are under debate: Influence of the orbital repulsion and/or formation of a defect band. In the present work, we applied a combination of the absorption and emission measurements on the samples exhibiting an outstanding nanoscale level of $(\mathrm{ZnO})_{1-x}(\mathrm{GaN})_{x}$ homogeneity as monitored by the high resolution electron microscopy equipped with the energy dispersive x-ray analysis and the electron energy loss spectroscopy; moreover the experimental data were set in the context of the computational analysis of the alloys employing density functional theory and quasiparticle $G W$ approximation. A prominent discrepancy in the band gap values as deduced from the absorption and emission experiments was observed systematically for the alloys with different compositions and interpreted as evidence for the absorption gap shrinking due to the defect band formation. Computational data support the argument, revealing only minor variations in the bulk of the conduction and valence band structures of the alloys, except for a characteristic "tail" in the vicinity of the valence band maximum. As such, we conclude that the energy gap bowing in $(\mathrm{ZnO})_{1-x}(\mathrm{GaN})_{x}$ alloys is due to the defect band formation, presumably at the top of the valence band maximum.
\end{abstract}

DOI: 10.1103/PhysRevB.100.165201

\section{INTRODUCTION}

Due to the band bowing effect, the optical absorption of the $(\mathrm{ZnO})_{1-x}(\mathrm{GaN})_{x}(\mathrm{ZOGN})$ alloy system can be tuned from the UV range well into the visible part of the spectrum [1,2]. This intriguing optical property makes the system interesting for photovoltaic and solar water-splitting applications. The band bowing effect occurs when the apparent band gap of the alloy is reduced as a function of composition, in a nonlinear fashion, with respect to the two unmodified materials. Various synthesis routes have been used to fabricate the ZOGN alloy, targeting the absorption tunability to meet the specific needs of different applications; for example nanowires were deposited using a sol-gel method [3] and high-temperature vapor-phase diffusion reactions [4], whereas pulsed-laser deposition [5] and magnetron sputtering [6] resulted in highly crystalline thin films. Solid solution synthesis was utilized to fabricate bulk samples for water-splitting applications [1,7-9]. In previous work, density functional theory (DFT) has been employed to investigate the origin of the band bowing effect observed for the ZOGN alloys; specifically repulsion between the $\mathrm{N} 2 p$ and $\mathrm{Zn} 3 d$ orbitals [10-13] and type II band alignment $[14,15]$ were suggested as possible explanations; in its turn Hirai et al. suggested that the absorption of visible light occurs via Zn-related acceptor levels [16].

While several theoretical explanations for the origin of the observed band bowing effect in the ZOGN have been discussed in the literature [10-15], experimental investigations of the mechanisms governing the band bowing effect are rather limited. Yang et al. combined transmission and reflection measurements with DFT calculations, finding that the absorption spectra do not match with the direct band gap Tauc plots and explained this observation with localization of the valence band maximum (VBM) on $\mathrm{N}$ atoms bonded with $\mathrm{Zn}$ [5]. Hirai et al. used photoluminescence (PL) in the wavelength range of 400-800 $\mathrm{nm}(3.1-1.55 \mathrm{eV})$ to argue that $\mathrm{Zn}$-acceptor levels in $\mathrm{GaN}$ are responsible for the absorption in the visible range [16]. However, the majority of the experimentally obtained band gap energies were extracted from transmission and/or reflectance measurements, while other type of band gap measurements employing complementary techniques have rarely been applied to ZOGN.

In the present work we investigate the band bowing effect in highly crystalline thin films of ZOGN deposited by magnetron sputtering. Band gaps were extracted using both photon absorption and electron energy loss spectra and compared to emission measurements. Furthermore, we use DFT together with the beyond-DFT hybrid functional HSE06 and quasiparticle $G W$ approximation to investigate the absorption, density of states (DOS), and band structure of the alloy in order to gain better understanding of the physical mechanisms responsible for the observed band bowing effect.

\section{EXPERIMENTAL DETAILS}

ZOGN thin films with different GaN content $x$ were synthesized using RF magnetron sputtering on $c$-plane $\mathrm{Al}_{2} \mathrm{O}_{3}$ substrates. During sputtering, the voltage was simultaneously applied to two separate targets: $\mathrm{ZnO}$ (99.99\% purity) and $\mathrm{GaN}\left(99.99 \%\right.$ purity). Both $\mathrm{Ar}$ and $\mathrm{N}_{2}$ were mixed in the 
chamber to tune the stoichiometry of the films. To obtain $x=0.02,30$ and $7 \mathrm{~W}$ were applied to the $\mathrm{ZnO}$ and $\mathrm{GaN}$ targets, respectively, while the Ar and $\mathrm{N}_{2}$ flows were set to 12.5 and $0.7 \mathrm{sccm}$, respectively. For $x=0.15$, the parameters were adjusted to $30 \mathrm{~W}(\mathrm{ZnO}), 20 \mathrm{~W}(\mathrm{GaN}), 12.5 \mathrm{sccm}$ (Ar), and $5 \mathrm{sccm}\left(\mathrm{N}_{2}\right)$ - for the summary of the synthesis details, see Table I in the Supplemental Material [17]. A substrate temperature at $400{ }^{\circ} \mathrm{C}$ and rotation at $11 \mathrm{rpm}$ was used for all depositions. The growth duration was adjusted to obtain film thickness of $\sim 1.5 \mu \mathrm{m}$. Post-deposition isochronal anneals at 600,700 , and $800^{\circ} \mathrm{C}$ in nitrogen atmosphere were employed to improve structural and optical properties. More detailed descriptions of the sample preparation, annealing, and structural characterization can be found elsewhere $[6,18]$.

The optical transmission measurements were carried out using the Shimadzu SolidSpec-3700 DUV spectrophotometer. The instrument utilizes a grating monochromator, as well as a range of detectors. The wavelength range of this instrument is $175-2600 \mathrm{~nm}$, with a resolution of $0.1 \mathrm{~nm}$. The measured transmittance curves were corrected for the spectral response of the instrument. The PL measurements were performed at $10 \mathrm{~K}$ keeping the samples inside a continuously pumped Janis Research helium refrigerator. The following continuous-wave excitation sources were used for the measurements: $\mathrm{He}-\mathrm{Cd}$ laser $(325 \mathrm{~nm}$, nominal optical power approximately $5 \mathrm{~mW}$, IK Series from Kimmon), Ar laser (488 nm, nominal optical power approximately $5 \mathrm{~mW}$, from Melles Griot), and solidstate laser $(405 \mathrm{~nm}$, nominal optical power approximately 5 or $100 \mathrm{~mW}$, from Oxxius). The PL emission was collected by a microscope, and afterwards either directed into the imaging spectrometer (Horiba Jobin Yvon, iHR320) coupled to a CCD camera (Luca DL-658M EMCCD from Andor), or into an optical fiber connected to a spectrometer (USB4000 from Ocean Optics) acting as two different detectors. The wavelength resolution in both cases was approximately 2-3 nm. Low-pass filters were used for the laser sources with excitation wavelengths of 488 and $405 \mathrm{~nm}$ in order to prevent scattered laser light from entering into the detection path. A correction scheme was used to transfer the measured data to PL intensity vs photon energy, instead of the PL intensity vs wavelength [19]. In addition, the PL spectra were also corrected for an exponential background originating from the scattered laser light entering the detection path even though low-pass filters were employed. PL data were collected as a function of excitation power using a neutral density filter wheel.

Samples were prepared for transmission electron microscopy (TEM) investigations as cross-section wedges by standard cutting, grinding, and polishing methods. Final sample thinning by Ar ion milling was done using the Gatan PIPS II. Prior to the TEM experiments, the samples were plasma cleaned using the Fishione Model 1020. TEM investigation, scanning TEM (STEM) imaging, energy dispersive $\mathrm{x}$-ray (EDX) analysis, and electron energy loss spectroscopy (EELS) were performed using a FEI Titan G2 60-300 kV TEM, equipped with a monochromator, a CEOS DCOR probe-corrector, and Super-X EDX detectors. All STEM imaging and EDX investigations were performed at an acceleration voltage of $300 \mathrm{kV}$ with a probe convergence angle of
$21 \mathrm{mrad}$ and a resulting spatial resolution of approximately $0.08 \mathrm{~nm}$. For band gap measurements in EELS an acceleration voltage of $60 \mathrm{kV}$ was used to reduce relativistic effects that might otherwise mask the band gap signal [20]. The spectra were acquired using a Gatan GIF 965 spectrometer, with a collection angle of approximately $20 \mathrm{mrad}$. A full with half maximum of the EELS zero-loss peak (ZLP) of $0.13 \mathrm{eV}$ was achieved, and the data for different samples were calibrated by aligning all ZLPs. Applying principal component analysis (PCA) [21] with 25 components, the noise in the EELS signal was reduced and the background was subtracted using a decaying power-law model [22].

\section{COMPUTATIONAL METHODS}

The computations of the ZOGN alloys were based on the DFT using the hybrid functional HSE06 and quasiparticle $G W$ approximation, i.e., the method employing the Green function and the screened Coulomb potential, using the VASP software package $[23,24]$. The hybrid functional HSE06 $[25,26]$ uses $25 \%$ Fock short-ranged exact exchange in conjunction with the exchange and full correlation energy from the local DFT potential. The $G W$ method relies on many-body selfconsistent calculations where the independent single particle equation contains a self-energy term. In our calculations, the partial self-consistent method scGW0 [27] was used, in which the screened dielectric function was kept constant while the Green functions were updated. A cut-off energy of $400 \mathrm{eV}$ was used in the first two steps for the DFT and HSE06 parts, and a cutoff of $100 \mathrm{eV}$ to describe the response function in the $G W$ part. A 72-atom unit cell structure was modeled for the alloys. This somewhat small cell is justified by the fact that we study alloys (and not defects), and that we employ computationally expensive techniques which have the advantages to reproduce interesting optical features as well as to generate band gap energies much closer to the experimental results. From this cell, seven $\mathrm{ZnO}$ pairs where randomly exchanged by $\mathrm{GaN}$ pairs, giving a concentration of $x=14 / 72=0.194 \approx 0.2$, as seen on the right-hand side in Fig. 1. Tests have been conducted with more than 30 different configurations. Within all the relaxed structures, the most thermodynamically stable
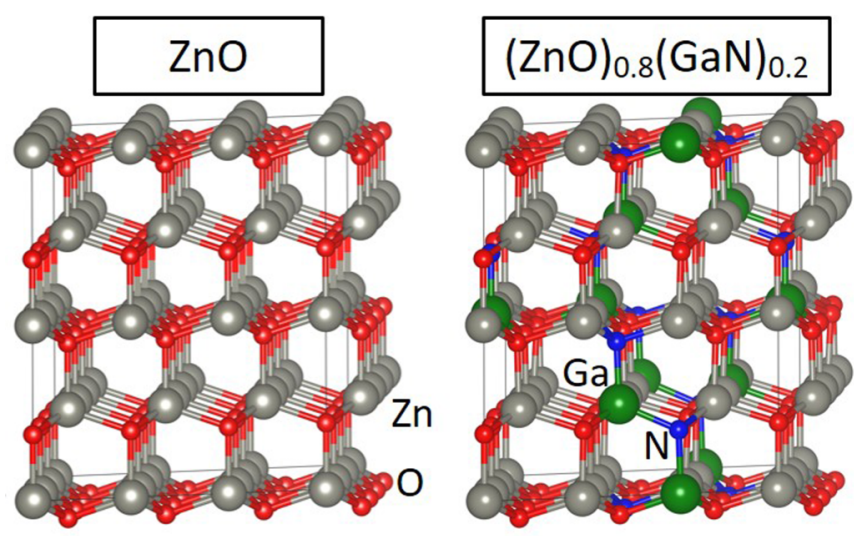

FIG. 1. Crystal structures for (left) binary $\mathrm{ZnO}$ and (right) for the most thermodynamic stable ZOGN alloy of the considered configurations with $x=7 / 36 \approx 0.2$. 
structures presented wider band gaps and more Ga-N bonds, with the final structures having an average close to $1.6 \mathrm{Ga}-\mathrm{N}$ bonds per GaN pair. A test with genetic algorithms [28] to find the best structure for the specific concentration, resulted in a configuration with total energy and band gap in the same range as the one presented. With this, only the most stable structure was selected for further simulation with the $\operatorname{sc} G W 0$ and the optical routines. Using a cut-off energy of $700 \mathrm{eV}$, the relaxation was done alternating between the volume and atom position relaxations, until the interaction remains $<5 \mathrm{meV} / \AA$. A $\Gamma$-centered $k$ mesh of $2 \times 2 \times 2$ was used in all calculations. The data for energy dispersion (Fig. 7) were obtained with the PBE functional [29], though gap energy has been corrected to the results from the $G W$ calculations. The optical properties were computed according to the linear response theory; the complex dielectric function were obtained from the optical matrix elements and the $G W$ energies for direct transitions from valence to conduction bands [30]. The optical spectra were calculated with a $25 \mathrm{mV}$ broadening. Our $G W$ calculations overestimate the band gap of pure $\mathrm{ZnO}$ with $\mathrm{E}_{g}(G W)=3.66 \mathrm{eV}$ which is $\sim 0.3 \mathrm{eV}$ higher experimental data; as such the calculated band gap energies were shifted to fit the experimental band gap of $\mathrm{ZnO}$ for better comparison (e.g., Figs. 3 and 8).

\section{RESULTS}

\section{A. Experimental results}

The deposited ZOGN films consist of a highly crystalline single domain with no secondary or amorphous phases present, as seen in Figs. S1(c) and S1(d) in the Supplemental Material [17]. In addition, the films form an atomically sharp interface with the $\mathrm{Al}_{2} \mathrm{O}_{3}$ substrate, as seen in Fig. S1(a). Furthermore, EDX analyses indicated a homogeneous random alloy without elemental clustering at the meso- and nanoscale, as can be seen in Fig. S2 in the Supplemental Material [17].

Figure 2 shows the measured photon absorption and electron energy loss data for ZOGN films with $x=0,0.02$, and 0.15 . Notably, the optical data in Fig. 2 are presented as Tauc plots assuming direct allowed transitions, while the EELS data are scaled linearly. A distinct shift towards lower energies is observed in Fig. 2 with a reference to $x=0$, i.e., pure $\mathrm{ZnO}$. Considering the optical data; $x=0.02$ and 0.15 results in onset shifts of $\sim 0.3$ and $\sim 0.8 \mathrm{eV}$, respectively, comparing to that of $\mathrm{ZnO}$. These shifts can readily be attributed to a reduction of the band gap of the alloy relative to the binary compositions. The evolution of the EELS data resembles that of the optical absorption, especially for $x=0$ and 0.15 , thereby confirming the trend for the band gap reduction in the alloys. The tail of the optical absorption onset is normally related to disorder, localized states, or compensation [31,32]. In Fig. 2 the tails are more prominent for the optical absorption compared to the electron energy loss; altogether it may indicate a significant amount of localized states inside the band gap, compensation, or disorder. This prominently holds specifically for $x=0.02$, where the optical absorption tail is significantly larger, indicating a high concentration of defects. Most importantly, the band gaps were estimated from the data in Fig. 2 (and similar data for $x=0.07,0.19,0.20$ not shown) and used in further analysis.

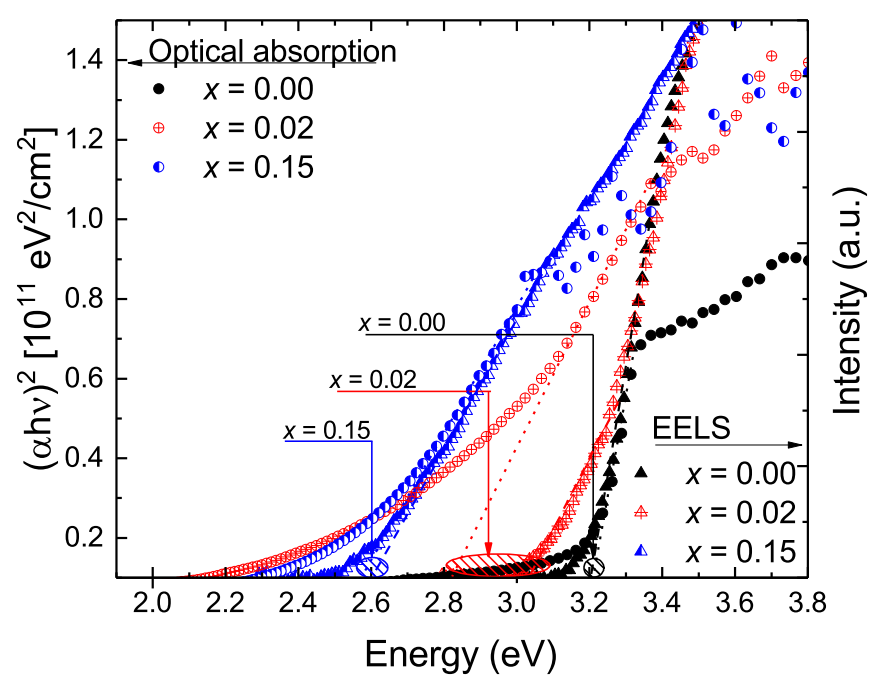

FIG. 2. Optical absorption and electron energy loss spectra for $x=0,0.02$, and 0.15 - see corresponding symbols in the legend. The optical data are presented in the form of Tauc plots assuming direct allowed transitions, while the EELS data are linearly scaled. Dotted (optical absorption) and dashed (EELS) lines represents a extrapolation of the linear region intersecting the $x$ axis. The point of intersection marks $E_{g}$.

The band gaps of the alloys as a function of composition are plotted in Fig. 3 together with the values calculated in the present study. In addition, the literature data are also included in Fig. 3 [2,8,9,33,34]. As shown in Fig. 3, a strong band gap reduction is observed at the initial stages of the alloying, terminated by a "plateau" at 2.6-2.4 eV. Notably, the calculated data predict a somewhat lower plateau of $(2.4-2.2 \mathrm{eV})$. The general trends unveiled in our measurement are in accordance with the literature data displayed in Fig. 3, even though the lower $x$ range has not been investigated

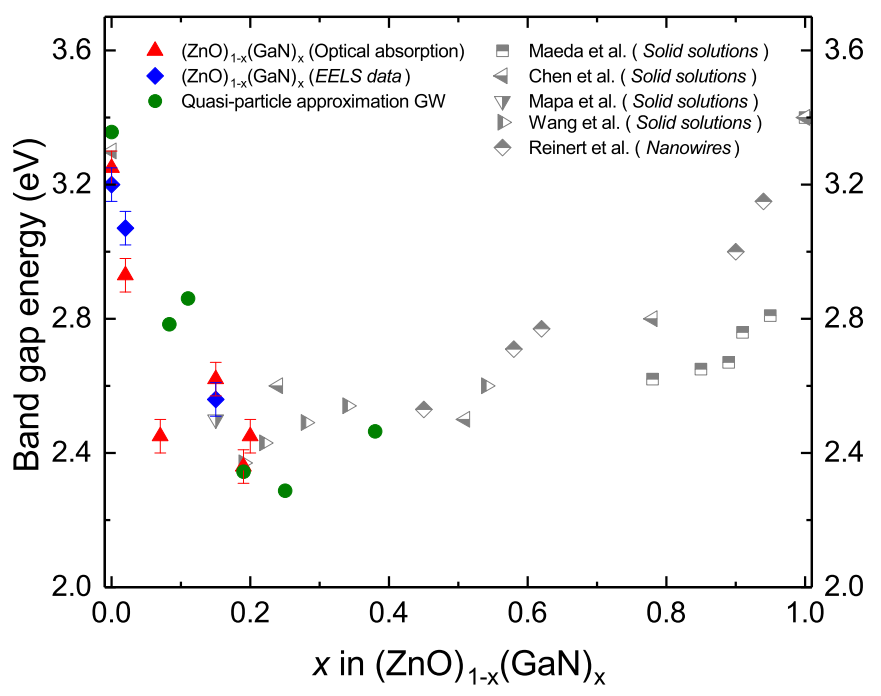

FIG. 3. The band gap magnitude as a function of $x$, as determined by the transmission measurements (filled triangles), STEM EELS (filled diamonds), and theory using $G W$ approximation (filled circles). The results are compared to the literature data (partly filled symbols) [2,8,9,33,34]. 


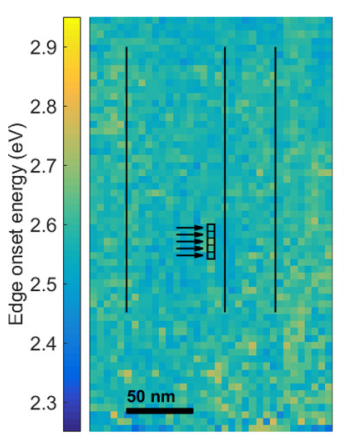

(a)

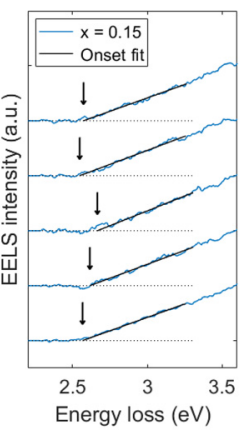

(b)

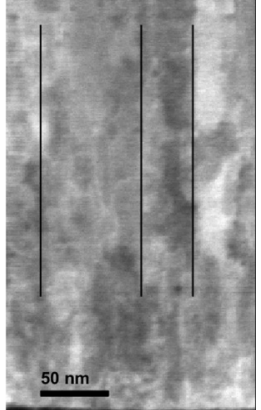

(c)
FIG. 4. STEM EELS data for $x=0.15$; (a) the band gap map, (b) EELS signal onset at specific pixels as indicated by arrows in (a), and (c) a dark-field image of the band gap map area. The vertical black lines [in (a) and (c)] are to emphasize the presence of the grain boundaries.

that much previously. The strong band gap reduction with increasing alloy composition is observed for both the $\mathrm{GaN}$ and $\mathrm{ZnO}$-rich sides of Fig. 3, revealing a relatively flat region in between. To divide the band bowing effect into these three regions of different band gap reduction, i.e., low (high)- $x$ regions with large $E_{g}$ reduction and a more $E_{g}$-stable region in between, has also been proposed as a model explaining the band bowing effect for $(\mathrm{ZnO})_{1-x}(\mathrm{~S})_{x}$ and $(\mathrm{ZnO})_{1-x}(\mathrm{Se})_{x}$ [35]. Opposing to the trends in Fig. 3, the $(\mathrm{ZnO})_{1-x}(\mathrm{~S})_{x}$ alloys have also been found to yield a gradual variation of the band gap through a wider range of compositions [36-38].

The optical absorption and the EELS data in Fig. 2 probe regions of $\sim 150 \mu \mathrm{m}$ in diameter and $200 \times 400 \mathrm{~nm}^{2}$, respectively. The EELS data can also be presented in the form of spatially resolved maps discriminating $\sim 5 \mathrm{~nm}$ pixels [39]. Figure 4 shows (a) an example of such map for $x=0.15$, (b) onset for a number of pixels as indicated by arrows in the map, and (c) corresponding dark-field image of the mapped area of the sample. As seen already from the EELS map itself, i.e., Fig. 4(a), the distribution of the signal is quite homogeneous, even over the columnar grain boundaries [marked by the vertical lines for clarity in Figs. 4(a) and 4(c)]. Moreover, plotting the signals from several pixels, see Fig. 4(b), demonstrates a close to uniform energy loss onset among the pixels, as indicated by the vertical arrows. The maximum variance over the whole of the mapped area is $\pm 0.25 \mathrm{eV}$. As such, these results indicate that the apparent band gap is the result of a homogeneous alloy, and not an average of a mixture of phases. Any such clustering or phase separation would have to be at length scales below $\sim 5-10 \mathrm{~nm}$. To further investigate potential cluster formation, STEM EDX analyses were performed. Figure 5 shows a nanoscale EDX map of an $26 \times 26 \mathrm{~nm}^{2}$ area and $0.4 \mathrm{~nm}$ pixel size for $x=0.15$. Figure 5 reveals a random alloy, although a small inhomogeneity in the compositional distribution was observed, specifically by averaging the signals of four pixels $\left(0.8 \times 0.8 \mathrm{~nm}^{2}\right)$, However, according to the calculated standard deviations, only chemical variations higher than $20 \%$ could be detectable with this method, which does not seem to be the case. It might seem somewhat surprising that Ga-N pairs will form a cluster, considering that the defect pairs are isovalent to the $\mathrm{ZnO}$ host, and the Ga-N bond length is very similar to that of $\mathrm{Zn}-\mathrm{O}$. For example, the relaxation effect is very small when incorporating GaN; see Fig. 1. Of course, the trust to find a perfectly random alloy configuration with only isolated Ga-N pairs is ambiguous, and somewhat smaller clusters may not be excluded. Meanwhile, it has been demonstrated that formation of neutral and valence-conserving larger complexes is also driven by the octet rule for the atoms [40]. Indeed, theory predicts that the volume allowed each atom neighboring to a Ga-N pair does not perfectly match the octet rule; so that it is slightly more favorable that two Ga-N pairs form a complex to minimize the total deviation from the octet rule in the alloy.

Furthermore, PL data collected at an excitation wavelength of $325 \mathrm{~nm}(3.82 \mathrm{eV})$ are presented in Fig. 6 for $x=0,0.02$, and 0.15 . All three samples reveal strong near-band emission (NBE) at 3.3-3.4 eV, corresponding to free- and acceptorbound excitons, exhibiting the highest intensity at $x=0.02$. The lower PL response for the $x=0.15$ sample can be due to experimental conditions, as well as the sample containing more nonradiative defects. The pure $\mathrm{ZnO}$ film differs from the two alloy compositions in showing excitonic features related to donor- and acceptor-bound excitons, potentially originating from $\mathrm{Ga}$ and $\mathrm{N}$ acting as dopants in $\mathrm{ZnO}$ [41]. Such contaminations may arise from the deposition chamber, due to cross contamination from previous depositions. However, quite surprisingly no significant redshift in this NBE is observed in
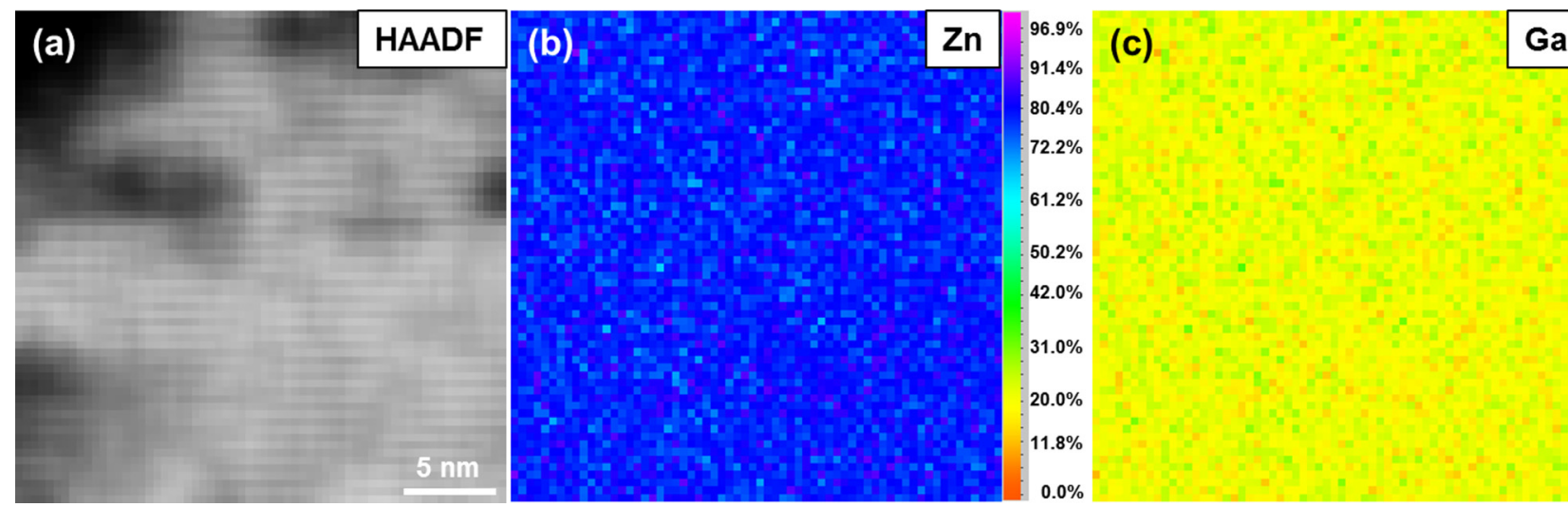

FIG. 5. (a) High-magnification HAADF-STEM image of the $x=0.15$ thin film and the corresponding EDX maps of (b) Zn and (c) Ga atomic percent. 

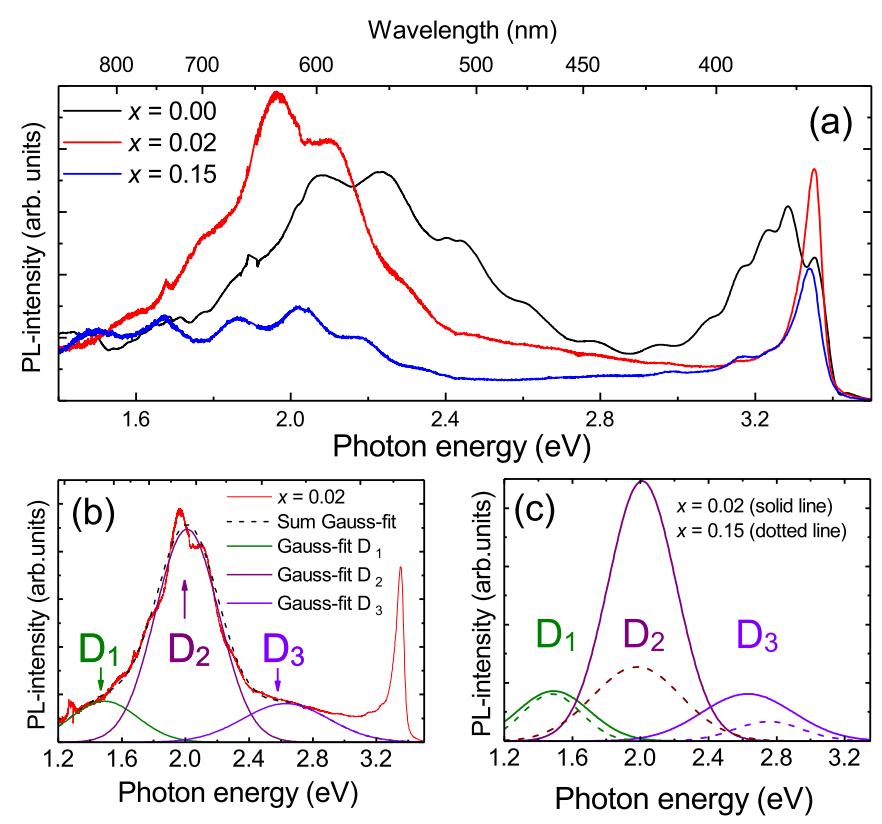

FIG. 6. (a) PL spectra recorded at $10 \mathrm{~K}$ for samples with different alloy compositions. (b) A deconvolution of the broad band emission part of the spectrum for $x=0.02$. (c) The deconvoluted contributions for $x=0.02$ (solid lines) and $x=0.15$ (dotted lines).

Fig. 6(a) as a function of $x$; which might be expected based on the data in Figs. 2-4, where the shifting onset in optical absorption and EELS data are interpreted in terms of a reduction in the band gap. Interestingly, the NBE still resembles that of pure $\mathrm{ZnO}$ films originating from excitonic transitions, unveiling no further structure compared to pure $\mathrm{ZnO}$. As it holds to the deep level emission of pure $\mathrm{ZnO}$, it shows a broad band between 1.8 and $2.6 \mathrm{eV}$, where contributions from native defects in $\mathrm{ZnO}$ including $\mathrm{V}_{\mathrm{O}}[42,43], \mathrm{O}_{i}$ [44], and $\mathrm{O}_{\mathrm{Zn}}$ [45], in addition to contributions from nitrogen-related defects, e.g., $\mathrm{N}_{\mathrm{O}}$ [46] are expected. In its turn, the deep level emission of the alloys is significantly altered and was found to be modeled by three Gaussian-like contributions. The deconvoluted contribution for $x=0.02$ are displayed in Fig. 6(b) revealing mean energy positions of 1.56-1.59 eV (D1), 1.81-1.98 eV (D2), and 2.23-2.46 eV (D3). The relative contributions of D1, D2, and D3 depends on $x$, as seen in Fig. 6(c). For example, the intensity of D1 is similar for both $x=0.02$ and 0.15 , whereas D2 is significantly more intense for the $x=0.02$ thin film. To identify the origin of these dominant contributions, the PL intensity was measured for different excitation powers; indeed the PL intensity is known to follow the power law: $I_{\mathrm{PL}} \sim P^{k}$, where $k>1$ for band-to-band or excitonic emission and $<1$ for emission related to defects [47]. Both D1 and D2 result in $k<1$ for the $x=0.02$ and $x=0.15$ thin film, indicating that these emissions are indeed defect related. The energy positions of D1-D3 were also measured as a function of excitation wavelength, using excitation wavelengths of $325 \mathrm{~nm}(3.82 \mathrm{eV}), 405 \mathrm{~nm}(3.06 \mathrm{eV})$, and $488 \mathrm{~nm}(2.54 \mathrm{eV})$ lasers, respectively (see Fig. S3 in the Supplemental Material [17]). Interestingly, the energy position of D1 was constant when increasing the excitation wavelength, whereas D2 and D3 shifted towards lower photon energies.
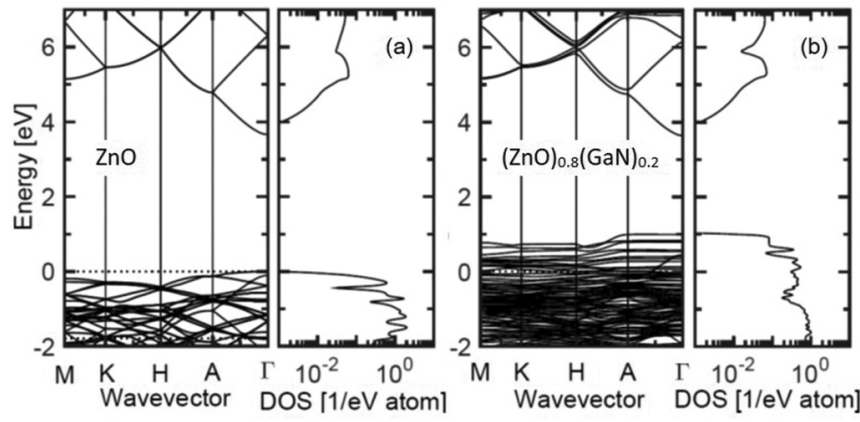

FIG. 7. Electronic band structures for (a) binary $\mathrm{ZnO}$ and (b) for the most thermodynamic stable ZOGN alloy with $x=7 / 36 \approx 0.2$. Notice that the density-of-states (DOS) is presented on a logarithmic scale in order to better visualize the states near the conduction band minimum. The energy zero scale is set at the VBM of binary $\mathrm{ZnO}$.

Summarizing experimental data, the evolution of the absorption onsets display a systematic and significant redshift, reaching $\sim 0.8 \mathrm{eV}$ at $x=0.15$, see Figs. $2-4$. This redshift was also found to be quite uniform over the sample, within the accuracy of the STEM EELS corresponding to $5 \mathrm{~nm}$. On the other hand, the PL near-band-edge emission resembles that of pure $\mathrm{ZnO}$, revealing no redshift as a function of the alloy composition. Meanwhile, the shapes of the PL near-band-edge and broad band emissions are quite different as a function of $x$.

\section{B. Theoretical calculations}

As mentioned above, the band gap energies obtained from our theoretical calculations are included in Fig. 3 (circles). These data represent estimates for the most stable configurations, however, not all possible configurations have been simulated and other configurations could potentially be more energetically favorable. Indeed, a strong band bowing effect, as seen from Fig. 3, is confirmed for $x \leqslant 0.25$; very importantly, in spite of significant experimental and theoretical uncertainties, the data are in a remarkable agreement.

The theoretical analysis of the electronic structure (see Fig. 7 showing the data for pure $\mathrm{ZnO}$ and ZOGN alloy with $x=7 / 36 \approx 0.2$ ) reveals a fairly similar band structures and DOS. The band gap energy of the alloy is smaller, but the conduction bands are very similar, resembling that of $\mathrm{ZnO}$. The main difference is the valence band structure, where the valence band maximum (VBM) of $x \approx 0.2$ [see Fig. 7(b)] lies $\sim 1 \mathrm{eV}$ above that of pure $\mathrm{ZnO}$ [see Fig. 7(a)]. The topmost bands become very flat in the alloy, especially in the (001) direction; this trend holds both for $\Gamma-A$ and $H-K$ symmetry lines. Therefore, the VBM for the $x \approx 0.2$ sample is at the $A$ point $[0,0,1]$, whereas the conduction band minimum (CBM) is a the $\Gamma$ point just like for pristine $\mathrm{ZnO}$.

The absorption coefficient $\alpha$ of the ZOGN alloy for $x \approx 0.2$ was calculated directly from the complex dielectric function: $\alpha(\omega)=\omega / c\left[2|\epsilon(\omega)|-2 \epsilon_{1}(\omega)\right]^{1 / 2}$. Figure 8 shows the calculated absorption spectra of several selected concentrations. As seen from Fig. 8, there are no features indicating localized defect states from the GaN pairs; instead it reveals a smooth absorption as an average of $\alpha$ of the $\mathrm{ZnO}$ and $\mathrm{GaN}$ binary 


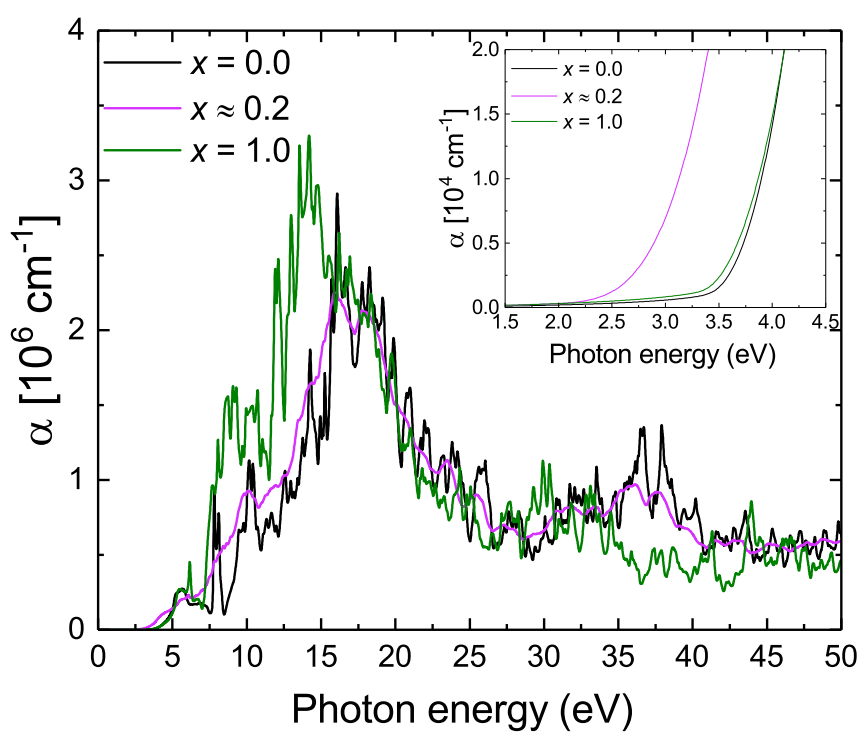

FIG. 8. Calculated absorption coefficients of ZOGN for $x=0$, 0.2 , and 1 , obtained with the $G W$ electronic structures. The inset shows the absorption coefficient in the region close to the band gap.

fractions. For the lower energy part of the data there are two main features: First, the remaining absorption peak around 5.5-6.5 eV present for both $\mathrm{ZnO}$ and $\mathrm{GaN}$; second, the spectrum for $x \approx 0.2$ is shifted by $\sim 1 \mathrm{eV}$ towards lower energies (see inset in Fig. 8). This combination of two features indicates that ZOGN has a similar electronic structure as its binary constituents, however the effective band gap is changed by $\sim 1 \mathrm{eV}$. From the electronic structure we know the energetically lowest energy transition for the $x \approx 0.2$ sample is the indirect transition from the highest occupied state at the $A$ point to the lowest empty state at the $\Gamma$ point. However, the energy for the indirect transition is only $30 \mathrm{meV}$ smaller than the direct transition at the $\Gamma$ point. While the optical transitions considers only direct transitions, the very flat topmost valence bands implies a stronger absorption near $E_{g}$. That is also seen in the absorption coefficient.

To further analyze the opposing results obtained from absorption and emission measurements (Figs. 2-4 versus Fig. 6), the partial atom-resolved density-of-states (PDOS) was calculated for $x \approx 0,0.02$, and 0.2 , where VBM of $x=0$ is used as reference. The orbital-projected PDOS is presented in the Supplemental Material [17] (Fig. S4). Figure 9 shows both the total DOS for $\mathrm{ZnO}$ compared to that of ZOGN, as well as the PDOS of the $\mathrm{Zn}+\mathrm{O}$ atoms and the $\mathrm{Ga}+\mathrm{N}$ atoms for the alloy, for $x \approx 0.02$ in Fig. 9(a) and $x \approx 0.2$ in Fig. 9(b). In the upper panel of Fig. 9(a), the $x \approx 0.02$ shows DOS above the VBM of binary $\mathrm{ZnO}$ (dotted vertical line), which from the lower panel can be seen to originate from $\mathrm{Ga}$ and $\mathrm{N}$ orbitals. What is obvious from the PDOS is that $\mathrm{Zn} d$-like states at about $-6 \mathrm{eV}$ are aligned between $\mathrm{ZnO}$ and $\mathrm{ZOGN}$. Also the main character of the $\mathrm{O} p$-like states are similar in $\mathrm{ZnO}$ and ZOGN. However, for the alloy, there is a clear contribution from rather localized $\mathrm{Ga}+\mathrm{N}$ states in the energy region near the VBM. This is a consequence of the energetically higher $\mathrm{N}$ $p$ states. The upper panel in Fig. 9(b) shows the total DOS for $\mathrm{ZnO}$ compared to that of $x \approx 0.2$, and the lower panel shows the PDOS for the $\mathrm{Zn}+\mathrm{O}$ orbitals and $\mathrm{Ga}+\mathrm{N}$ orbitals for the alloy. For this composition the DOS for the alloy is more intense and broader in the energy region above the VBM of $\mathrm{ZnO}$ (dotted vertical line). From the semicore states below $-10 \mathrm{eV}$ in Fig. 9(b) we estimate roughly the valence band offset (VBO) to be about $1 \mathrm{eV}$, which is expected since $\mathrm{N}$ $p$ states are typically $1 \mathrm{eV}$ higher in energy than $\mathrm{O} p$ states $[48,49]$. Again, there is a pronounced contribution from rather localized $\mathrm{Ga}+\mathrm{N}$ states in the energy region near the VBM,
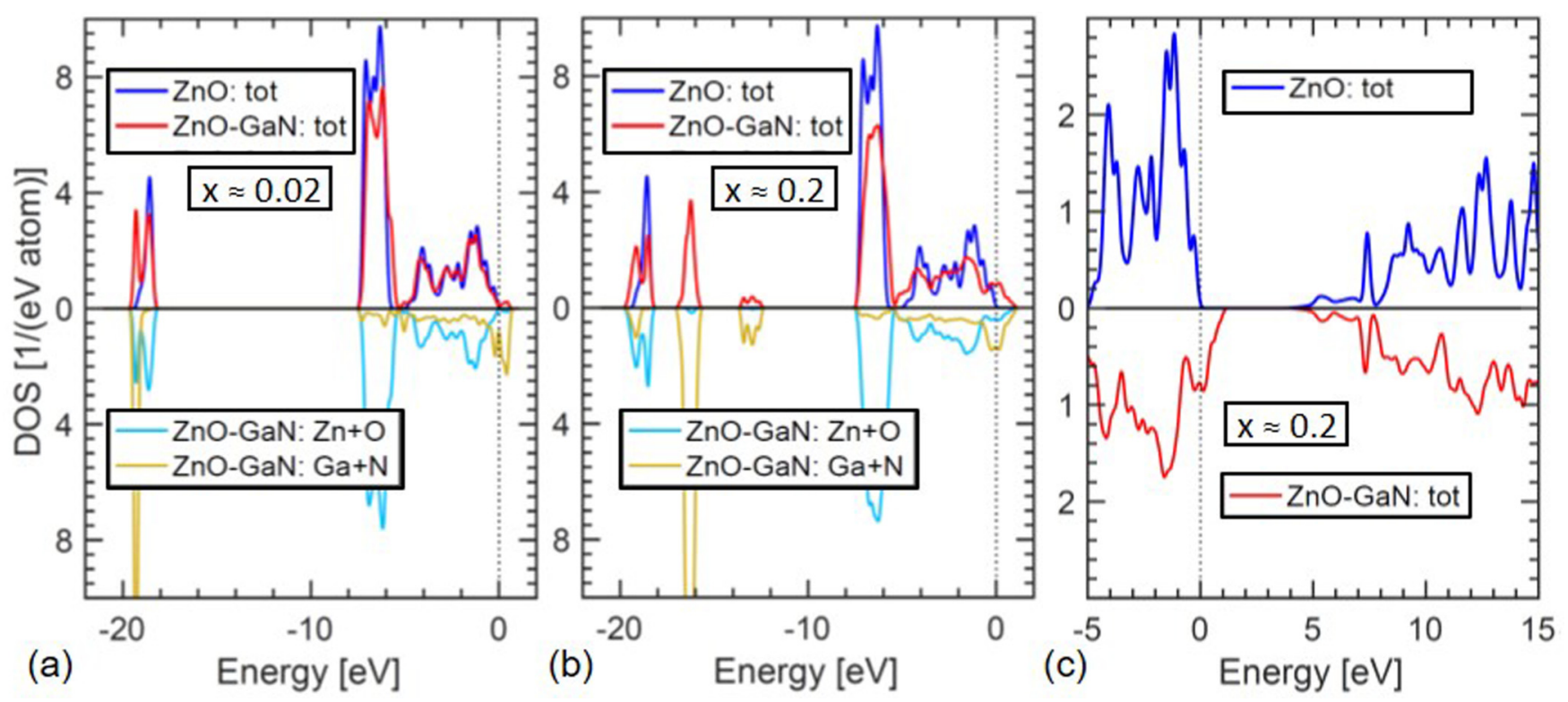

FIG. 9. (a) Total DOS of the valence and semicore states for $x=0$ (blue) and $x \approx 0.02$ (red) in the upper panel, whereas the PDOS from $\mathrm{Zn}$ and $\mathrm{O}$ atoms (light blue) and $\mathrm{Ga}$ and $\mathrm{N}$ atoms (orange) in the $x \approx 0.02$ sample are shown in the lower panel. (b) Same as in (a) but for $x \approx 0.2$. (c) Total DOS for $x=0$ (blue) and $x \approx 0.2$ (red), scaled to lower energies, in order to emphasize that the effect on the electronic structure due to GaN incorporation occurs mainly near the VBM. The dotted line indicates the VBM of pure ZnO. 
originating from the energetically higher $\mathrm{N} p$ states, also observed for $x \approx 0.02$. These localized states explain why the topmost valence bands have the flat character (Fig. 7). A similar result has also been observed for the $\mathrm{ZnO}_{1-x} \mathrm{~S}_{x}$ system, where the VBM has been found to consist of mainly $\mathrm{Zn}$ and $\mathrm{S}$ for $x>0$, where $\mathrm{S} p$ states are energetically higher than the $\mathrm{O} p$ states [36].

In Fig. 9(c) the total DOS for $x=0$ and $x \approx 0.2$ are again shown, but for the energy region relevant for optical absorption. With the VBO of about $1 \mathrm{eV}$, the main DOS peaks in both the valence bands and the conduction bands coincide for both compounds. The CBM is roughly at the same energy, and thus the band gap reduction of about $1 \mathrm{eV}$ in the ZOGN alloy is caused by an upward shift in VBM. This gap reduction is in good agreement with our experimental results in Fig. 3 and our calculated absorption in Fig. 8.

\section{DISCUSSION}

The data in Sec. IV showed that a strong band bowing effect is observed in the ZOGN alloy, even at relatively modest Ga-N pair concentrations. Several theories have been proposed to explain the origin of the band bowing effect, while the two prevailing explanations are (i) the alloy valence band is pushed upward in energy due to orbital repulsion, and (ii) a defect band is formed within the band gap of the binary compound, effectively reducing the apparent optical band gap of the alloy. Starting with (i), one would expect that a shift of the VBM towards higher energies (upward) would cause the near-band-edge emission (NBE) measured in PL to occur at lower energies compared to that of binary $\mathrm{ZnO}$, i.e., a gradual change in the energetic position of the NBE from that of pure $\mathrm{ZnO}$ towards lower energies. However, independently of $x$, the NBE remain similar to that of binary $\mathrm{ZnO}$ (Fig. 6). Furthermore, for other alloy materials with a strong band bowing effect, and where an upward shift of the VBM is expected to cause the band bowing, e.g., in $\mathrm{ZnO}_{1-x} \mathrm{~S}_{x}$ alloys [36], a more gradual change in the band gap is observed [36] upon alloying, in contrast to the present results for ZOGN. Meanwhile, the explanation in terms of the hypothesis (ii), is in a better agreement with the experimental observations; indeed the defect band would not alter the NBE observed in $\mathrm{PL}$, since the $\mathrm{ZnO}$ VBM to CBM transitions would remain a viable route for emission. Similar arguments can be put forth for the broad band emission, since the introduction of a defect band may not affect the energy position of the defect levels, opposite to what has been reported for defect levels following an upwards shift in VBM [36]. The fact that the energy position of the contributions D1-D3 remains unchanged in Fig. 6 is an additional argument in favor of (ii). Furthermore, the strong shift in the apparent band gap from $\sim 3.3$ to $\sim 2.4$ is in accordance with the introduction of a band with a specific energy arising in the material, where the apparent band gap absorption is related to a transition between conduction and defect bands. This further corroborates why no change in the absorption onset with increasing $\mathrm{GaN}$ content of the alloy is seen over the so called plateau in Fig. 3.

The theoretical calculations show a similar strong band gap reduction at modest $\mathrm{GaN}$ incorporations (i.e., below 25\%), although not as rapid as the experimental measurements indicate. Figure 9 shows that the states at the VBM in the alloy composition mainly consist of states formed by $\mathrm{N} p$-like orbitals, and that these DOS shift upward in energy and broaden, when increasing $x$ from 0.02 to 0.2 . Thus, $\mathrm{N}$ incorporation introduces defect states at a higher energy compared to that of binary $\mathrm{ZnO}$ (mainly arising from the $\mathrm{O} p$ orbitals), which can be interpreted as nitrogen-related defects forming a band at higher energy than the VBM of $\mathrm{ZnO}$, in accordance with our experimental observations. Figure 7 display the calculated band structure of the binary $\mathrm{ZnO}$ and the ZOGN alloy with $x \approx 0.2$, normalized to the VBM. From Fig. 7 one can observe a significantly reduced dispersion of the valence band of the alloy compared to the binary $\mathrm{ZnO}$, especially in the $\Gamma-A$ direction. The reduced dispersion arises since states are not required to be degenerate at the $A$ point for the alloy with lower crystallographic symmetry. Moreover, Fig. 7 indicates that an indirect transition has the lowest energy, as stated previously, with the top of the valence band at the $A$ point.

\section{CONCLUSION}

In conclusion, a significant redshift in both optical absorption and electron energy loss spectra is observed for the highly crystalline ZOGN thin films with $x \leqslant 0.20$. On the other hand, the measured near-band-edge emissions show no indication of the corresponding redshifts, still resembling what is expected for pure $\mathrm{ZnO}$, as a result of acceptor-bound and free exciton interactions. This discrepancy is interpreted in terms of the defect band formed within the band gap, and being responsible for the absorption within the visible spectrum in the alloys. The theoretical arguments were in agreement with the experimental facts.

\section{ACKNOWLEDGMENTS}

This work is funded by The Research Council of Norway (RCN) and is acknowledged for the support to the SALIENT project, Project No. 239895/F20. RCN is also acknowledged for the projects PVLife, Project No. 243642 and FUNDAMENT, Project No. 251131. RCN is also acknowledged for the support to the Norwegian Micro- and Nano-Fabrication Facility, NorFab, Project No. 245963/F50 and the Norwegian Center for Transmission Electron Microscopy, NORTEM, Project No. 197405/F50. We acknowledge access to highperformance computing resources through the Norwegian and Swedish infrastructures NOTUR and SNIC, as well as through DECI within the Partnership for Advanced Computing in Europe.
[1] K. Maeda, K. Teramura, T. Takata, M. Hara, N. Saito, K. Toda, Y. Inoue, H. Kobayashi, and K. Domen, Overall water splitting on $\left(\mathrm{Ga}_{1-x} \mathrm{Zn}_{x}\right)\left(\mathrm{N}_{1-x} \mathrm{O}_{x}\right)$ solid solution photocatalyst: Relationship between physical properties and photocatalytic activity, J. Phys. Chem. B 109, 20504 (2005). 
[2] A. A. Reinert, C. Payne, L. Wang, J. Ciston, Y. Zhu, and P. G. Khalifah, Synthesis and characterization of visible light absorbing $(\mathrm{GaN})_{1-x}(\mathrm{ZnO})_{x}$ semiconductor nanorods, Inorg. Chem. 52, 8389 (2013).

[3] W.-Q. Han, Y. Zhang, C.-Y. Nam, C. T. Black, and E. E. Mendez, Growth and electronic properties of $\mathrm{GaN} / \mathrm{ZnO}$ solid solution nanowires, Appl. Phys. Lett. 97, 083108 (2010).

[4] M. Zhong, Y. Ma, P. Oleynikov, K. Domen, and J.-J. Delaunay, A conductive $\mathrm{ZnO}-\mathrm{ZnGaON}$ nanowire-array-on-a-film photoanode for stable and efficient sunlight water splitting, Energy Environ. Sci. 4, 1693 (2014).

[5] C. Yang, Y. Hirose, T. Wakasugi, N. Kashiwa, H. Kawai, K. Yamashita, and T. Hasegawa, Epitaxy of $(\mathrm{GaN})_{1-x}(\mathrm{ZnO})_{x}$ Solid-Solution Thin Films with Widely Tunable Chemical Composition and Strong Visible Absorption, Phys. Rev. Appl. 10, 044001 (2018).

[6] V. S. Olsen, C. Bazioti, A. Azarov, B. G. Svensson, A. Kuznetsov, Ø. Prytz, and L. Vines, Bandgap bowing in crystalline $(\mathrm{ZnO})_{1-x}(\mathrm{GaN})_{x}$ thin films; influence of composition and structural properties, Semicond. Sci. Technol. 34, 015001 (2019).

[7] K. Maeda and K. Domen, Solid solution of GaN and $\mathrm{ZnO}$ as a stable photocatalyst for overall water splitting under visible light, Chem. Mater. 22, 612 (2010).

[8] H. Chen, L. Wang, J. Bai, J. C. Hanson, J. B. Warren, J. T. Muckerman, E. Fujita, and J. A. Rodriguez, In situ XRD studies of $\mathrm{ZnO} / \mathrm{GaN}$ mixtures at high pressure and high temperature: Synthesis of Zn-rich $\left(\mathrm{Ga}_{1-x} \mathrm{Zn}_{x}\right)\left(\mathrm{N}_{1-x} \mathrm{O}_{x}\right)$ photocatalysts, J. Phys. Chem. C 114, 1809 (2010).

[9] M. Mapa, K. S. Thushara, B. Saha, P. Chakraborty, C. M. Janet, R. P. Viswanath, C. M. Nair, K. V. G. K. Murty, and C. S. Gopinath, Electronic structure and catalytic study of solid solution of $\mathrm{GaN}$ in $\mathrm{ZnO}$, Chem. Mater. 21, 2973 (2009)

[10] J. Liu, M. V. Fernández-Serra, and P. B. Allen, Special quasiordered structures: Role of short-range order in the semiconductor alloy $(\mathrm{GaN})_{1-x}(\mathrm{ZnO})_{x}$, Phys. Rev. B 93, 054207 (2016)

[11] L. L. Jensen, J. T. Muckerman, and M. D. Newton, Firstprinciples studies of the structural and electronic properties of the $\left(\mathrm{Ga}_{1-x} \mathrm{Zn}_{x}\right)\left(\mathrm{N}_{1-x} \mathrm{O}_{x}\right)$ solid solution photocatalyst, J. Phys. Chem. C 112, 3439 (2008).

[12] S. H. Wei and A. Zunger, Role of $d$ states in II-VI semiconductors, Phys. Rev. B 37, 8958 (1988).

[13] C. D. Valentin, Electronic structure of $\left(\mathrm{Ga}_{1-x} \mathrm{Zn}_{x}\right) \mathrm{N}_{1-x} \mathrm{O}_{x}$ photocatalyst for water splitting by hybrid hartree-fock density functional theory methods, J. Phys. Chem. C 114, 7054 (2010).

[14] M. N. Huda, Y. Yan, S.-H. Wei, and M. M. Al-Jassim, Electronic structure of $\mathrm{ZnO}$ : GaN compounds: Asymmetric bandgap engineering, Phys. Rev. B 78, 195204 (2008).

[15] S. Wang and W. Lin-Wang, Atomic and Electronic Structures of GaN/ZnO Alloys, Phys. Rev. Lett. 104, 065501 (2010).

[16] T. Hirai, K. Maeda, M. Yoshida, J. Kubota, S. Ikeda, M. Matsumura, and K. Domen, Origin of visible light absorption in GaN-Rich $\left(\mathrm{Ga}_{1-x} \mathrm{Zn}_{x}\right)\left(\mathrm{N}_{1-x} \mathrm{O}_{x}\right)$ photocatalysts, J. Phys. Chem. C 111, 18853 (2007).

[17] See Supplemental Material at http://link.aps.org/supplemental/ 10.1103/PhysRevB.100.165201 for a full survey table of all samples prepared for this study. In addition, high-resolution STEM images, STEM EDX analyses, TEM selected area diffraction patterns are presented to address the crystal quality and relative elemental composition. The deconvolution of PL spectra for $x=0.15$ is shown, as well as the orbital-projected PDOS for $\mathrm{ZnO}, \mathrm{Zn}$ and $\mathrm{O}$ atoms in ZOGN $x \approx 0.2$, and $\mathrm{Ga}$ and $\mathrm{N}$ atoms for ZOGN $x \approx 0.2$.

[18] V. S. Olsen, C. Bazioti, G. Baldissera, A. Azarov, O. Prytz, C. Persson, B. G. Svensson, A. Y. Kuznetsov, and L. Vines, Effects of substrate and post-deposition annealing on structural and optical properties of $(\mathrm{ZnO})_{1-x}(\mathrm{GaN})_{x}$ films, Phys. Status Solidi (b) 256, 1800529 (2019).

[19] J. R. Lakowicz, Principles of Fluorescence Spectroscopy, 2nd ed. (Kluwer Academic/Plenum, Amsterdam, 1999).

[20] W. Zhan, C. S. Granerød, V. Venkatachalapathy, K. M. H. Johansen, I. J. T. Jensen, A. Yu. Kuznetsov, and Ø. Prytz, Nanoscale mapping of optical band gaps using monochromated electron energy loss spectroscopy, Nanotechnology 28, 105703 (2017).

[21] M. Bosman, M. Watanbe, D. T. L. Alexander, and V. J. Keast, Mapping chemical and bonding information using multivariate analysis of electron energy-loss spectrum images, Ultramicroscopy 106, 1024 (2006).

[22] C. S. Granerød, W. Zhan, and O. Prytz, Automated approaches for band gap mapping in STEM-EELS, Ultramicroscopy 184, 39 (2018).

[23] P. E. Blöchl, Projector augmented-wave method, Phys. Rev. B 50, 17953 (1994).

[24] G. Kresse and D. Joubert, From ultrasoft pseudopotentials to the projector augmented-wave method, Phys. Rev. B 59, 1758 (1999).

[25] J. Heyd, G. E. Scuseria, and M. Ernzerhof, Hybrid functionals based on a screened Coulomb potential, J. Chem. Phys. 118, 8207 (2003).

[26] A. V. Krukau, O. A. Vydrov, A. F. Izmaylov, and G. E. Scusiera, Influence of the exchange screening parameter on the performance of screened hybrid functionals, J. Chem. Phys. 125, 224106 (2006).

[27] M. Shishkin, M. Marsman, and G. Kresse, Accurate Quasiparticle Spectra from Self-Consistent $G W$ Calculations with Vertex Corrections, Phys. Rev. Lett. 99, 246403 (2007).

[28] L. Vilhelmsen and B. Hammer, A generic algorithm for first principles global structure optimization of supported nano structures, J. Chem. Phys. 141, 044711 (2014).

[29] J. P. Perdew, K. Burke, and M. Ernzerhof, Generalized Gradient Approximation Made Simple, Phys. Rev. Lett. 77, 3865 (1996).

[30] M. Gajdoš, K. Hummer, G. Kresse, J. Furthmüller, and F. Bechstedt, Linear optical properties in the projector-augmented wave methodology, Phys. Rev. B 73, 045112 (2006).

[31] E. O. Kane, Band tails in semiconductors, Solid-State Electron. 28, 3 (1985).

[32] J. K. Katahara and H. W. Hillhouse, Quasi-Fermi level splitting and sub-bandgap absorptivity from semiconductor photoluminescence, J. Appl. Phys. 116, 173504 (2014).

[33] K. Maeda, K. Teramura, D. Lu, T. Takata, N. Saito, Y. Inoue, and K. Domen, Photocatalyst releasing hydrogen from water, Nature (London) 440, 295 (2006).

[34] J. P. Wang, B. Huang, Z. Wang, P. Wang, H. Cheng, Z. Zheng, X. Qin, X. Zhang, Y. Dai, and M.-H. Whangbo, Facile 
synthesis of $\mathrm{Zn}$-rich $(\mathrm{GaN})_{1-x}(\mathrm{ZnO})_{x}$ solid solutions using layered double hydroxides as precursors, J. Mater. Chem. 21, 4562 (2011).

[35] G. Baldissera and C. Persson, Understanding the optical properties of $\mathrm{ZnO}_{1-x} \mathrm{~S}_{x}$ and $\mathrm{ZnO}_{1-x} \mathrm{Se}_{x}$ alloys, J. Appl. Phys. 119, 045704 (2016).

[36] C. Persson, C. Platzer-Björkman, J. Malmström, T. Törndahl, and M. Edoff, Strong Valence-Band Offset Bowing of $\mathrm{ZnO}_{1-x} \mathrm{~S}_{x}$ Enhances $p$-Type Nitrogen Doping of ZnO-like Alloys, Phys. Rev. Lett. 97, 146403 (2006).

[37] B. K. Meyer, A. Polity, B. Farangis, Y. He, D. Hasselkamp, T. Krämer, and C. Wang, Structural properties and bandgap bowing of $\mathrm{ZnO}_{1-x} \mathrm{~S}_{x}$ thin films deposited by reactive sputtering, Appl. Phys. Lett. 85, 4929 (2004).

[38] R. R. Thankalekshmi and A. C. Rastogi, Structure and optical band gap of $\mathrm{ZnO}_{1-x} \mathrm{~S}_{x}$ thin films synthesized by chemical spray pyrolysis for application in solar cells, J. Appl. Phys. 112, 063708 (2012).

[39] C. S. Granerød, A. Galeckas, K. M. Johansen, L. Vines, and O. Prytz, The temperature-dependency of the optical band gap of $\mathrm{ZnO}$ measured by electron energy-loss spectroscopy in a scanning transmission electron microscope, J. Appl. Phys. 123, 145111 (2018).

[40] D. Huang and C. Persson, Band gap change induced by defect complexes in $\mathrm{Cu}_{2} \mathrm{ZnSnS}_{4}$, Thin Solid Films 535, 265 (2013).

[41] L. Vines and A. Y. Kuznetsov, Oxide Semiconductors (Semiconductor and Semimetals, 2013), Vol. 88, pp. 67-99.
[42] T. M. Børseth, B. G. Svensson, A. Y. Kuznetsov, P. Klason, Q. X. Zhao, and M. Willander, Identification of oxygen and zinc vacancy optical signals in $\mathrm{ZnO}$, Appl. Phys. Lett. 89, 262112 (2006).

[43] D. M. Hofmann, D. Pfisterer, J. Sann, B. K. Meyer, R. TenaZaera, V. Munoz-Sanjose, T. Frank, and G. Pensl, Properties if the oxygen vacancy in $\mathrm{ZnO}$, Appl. Phys. A 88, 147 (2007).

[44] X. L. Wu, G. G. Siu, C. L. Fu, and H. C. Ong, Photoluminescence and cathodoluminescence studies of stoichiometric and oxygen-deficient $\mathrm{ZnO}$ films, Appl. Phys. Lett. 78, 2285 (2001).

[45] B. Lin and Z. Fu, Green luminescent center in undoped zinc oxide films deposited on silicon substrates, Appl. Phys. Lett. 79, 943 (2001).

[46] M. C. Tarun, M. Z. Iqbal, and M. D. McCluskey, Nitrogen is a deep acceptor in ZnO, AIP Adv. 1, 022105 (2011).

[47] T. Schmidt, K. Lischka, and W. Zulehner, Excitation-power dependence of the near-band-edge photoluminescence of semiconductors, Phys. Rev. B 45, 8989 (1992).

[48] T. D. Veal, P. D. C. King, S. A. Hatfield, L. R. Bailey, C. F. McConville, B. Martel, J. C. Moreno, E. Frayssinet, F. Semond, and J. Zúñiga-Pérez, Valence band offset of the $\mathrm{ZnO} / \mathrm{AIN}$ heterojunction determined by x-ray photoemission spectroscopy, Appl. Phys. Lett. 93, 202108 (2008).

[49] F. Oba and Yu. Kumagai, Design and exploration of semiconductors from first principles: A review of recent advances, Appl. Phys. Express 11, 060101 (2018). 